\title{
Statistical Effects in the Solar Activity Cycles during AD 1823-1996
}

\author{
Maxim Ogurtsov ${ }^{1}$ and Markus Lindholm ${ }^{2}$ \\ ${ }^{1}$ A.F. Ioffe Physico-Technical Institute, St. Petersburg 194021, Russia \\ ${ }^{2}$ Metla, Eteläranta 55, FI-96301, Rovaniemi, Finland \\ Correspondence should be addressed to Maxim Ogurtsov, maxim.ogurtsov@mail.ioffe.ru
}

Received 10 February 2011; Accepted 8 March 2011

Academic Editors: P. P. Avelino and M. Richmond

Copyright (C) 2011 M. Ogurtsov and M. Lindholm. This is an open access article distributed under the Creative Commons Attribution License, which permits unrestricted use, distribution, and reproduction in any medium, provided the original work is properly cited.

General statistical properties of solar activity cycles during the period AD 1823-1996-including the Gnevyshev-Ohl and Waldmeier effects as well as an amplitude-period effect—were analyzed using Wolf number, group sunspot number, and extended total sunspot area series. It was found out that the Gnevyshev-Ohl effect GO2 (the positive correlation between intensity of the even cycles $2 N$ and intensity of the odd cycles $2 N+1$ ) and the Waldmeier effect W2 (the anticorrelation between rise times of sunspot cycles and their amplitudes) are the most universal and robust features of the solar cycle. Other statistical relations were found appreciably sensitive to the selection of solar index, the interval of analysis, and the way of the cycle feature determination.

\section{Introduction}

The presence of a link between the Sun's activity and processes in circumterraneous space (atmosphere and magnetosphere), which form space weather, has been reliably established (see $[1,2])$. Space weather, in turn, considerably influences many aspects of human activity including radio communication and navigation as well as the functioning of energy supply networks. Thus, increasing our knowledge about solar variability and its general statistical characteristics is important not only for further development of the solar dynamo model, but also such knowledge has appreciable practical value. The 11-year cycle is the major characteristic of solar activity. In addition, sunspot cycles exhibit several significant statistical features, including the Gnevyshev-Ohl (even-odd) effect, Waldmeier effect, and an amplitude-period effect. These statistical relations have attracted attention of many researchers-see, for example, Vitinsky et al. [3], Charbonneau [4], Hathaway et al. [5], Dikpati et al. [6], Cameron and Schüssler [7], and Karak and Choudhuri [8]. Hathaway et al. [5] showed that in spite of some differences, these features are manifested throughout 1755-1996 (cycles 1-22 in Zürich numbering) in both Wolf (Zürich) sunspot number $R_{\mathrm{Z}}$ and group sunspot number $R_{\mathrm{G}}$ as introduced by Hoyt and Schatten [9]. However, sunspot numbers are purely statistical indices, which are determined from data of instrumental observations of the Sun using special mathematical algorithms. The Wolf number $R_{Z}$ is defined as

$$
R_{Z}=k(10 G+F)
$$

where $G$ is the number of identified sunspot groups, $F$ is the total number of individual sunspots, and the correction factor $k$ accounts for observational techniques and instrumentation at the individual observatory. Hoyt and Schatten [9] have introduced a new index of solar activity called the group sunspot numbers $R_{G}$. Group sunspot number is given by

$$
R_{G}=\frac{12.08}{N} \sum_{i=1}^{N} k_{i} G_{i},
$$

where $N$ is the number of observers, $k_{i}$ is the correction factor for observer $i, G_{i}$ is the number of sunspot groups reported by observer $i$, and 12.08 is a normalization factor scaling $R_{G}$ to $R_{Z}$ values for the period of 1874-1976 when Greenwich observatory provided daily reports on the number and 
characteristics of sunspot groups. As the sunspot number has no direct physical meaning, it is obviously important to test the robustness of the three types of statistical effects using some physical solar index, which quantifies the actual solar magnetic phenomena. Sunspot area could plausibly serve as such a physical index, since it is directly linked to the solar magnetic flux emerging at sunspots [10]. According to Nagovitsyn [10] this connection is described by

$$
\Phi_{\Sigma}(t) \approx 2.49 \times 10^{19} A(t),
$$

where $\Phi(t)_{\Sigma}$-magnetic flux in Ms, $A(t)$ - sunspot area in microsolar hemisphere.

Dikpati et al. [6] analyzed the Waldmeier effect (anticorrelation between the peak in sunspot number of a cycle and the rise time) in sunspot area for cycles 12-22 (18781996) using mainly data of the Royal Greenwich Observatory. They found out that the Waldmeier effect is not present in the spot area data; that is, the corresponding coefficient of linear correlation $R_{l}$ is close to zero. Moreover, Dikpati et al. [6] report that the statistical characteristics of the cycles in sunspot area are not identical to that of sunspot number. Karak and Choudhuri [8], however, presented some evidence for Waldmeier relations in different solar indices including sunspot area. These authors reported that coefficient of correlation between rise times of sunspot area cycles and their strengths $R_{l}=-0.31$. The difference most likely arises from different ways of the determination of the cycle rise time. It is known that it is not easy to establish the actual times of starting and ending points of the solar cycle, since two consecutive cycles overlap. Dikpati et al. [6] and Karak and Choudhuri [8] performed an analysis only for 12 cycles, since they were limited with Greenwich data available for the solar cycles 12-23. Recently, the Greenwich sunspot area series has been substantially expanded by astronomers from Pulkovo Observatory [11], who used additional information obtained by Schwabe, Carrington, Spörer, and de la Rue before 1874 and Soviet-Russian observers after 1976. Nagovitsyn et al. [11] constructed a monthly sunspot area data set covering the time interval 1821-2005 and calibrated it against the Greenwich general system (http://www.gao.spb.ru/database/esai/). The use of Pulkovo sunspot area series makes it possible to extend statistical analysis towards the cycles 7-11 (1823-1874) and to examine statistical features of the cycles in sunspot area over a substantially longer time covering 17 solar cycles. Joint analysis of the cycles in sunspot area, $R_{Z}$ and $R_{G}$, through the interval AD 1823-1996 is the main goal in the present work. Information on sunspot number over the selected time interval, 1823-1996, can be generally assessed as being quite reliable. Systematic uncertainties of $R_{G}$ values are less than 5\% over 1800-1849 and about 1\% since 1849 [9]. Eddy [12] considered Wolf numbers $R_{Z}$ reliability as good during 18181847 and fully reliable after 1848 .

\section{General Statistical Effects of Solar Cycles}

The Gnevyshev-Ohl (even-odd) rule $[3,13]$ reflects correlation of adjacent 11-year cycles and includes a few statements. The most widely used are the following. (a) The amplitude (maximum value over the cycle) of the odd-numbered solar cycle $2 N+1$ correlates positively with the amplitude of the even-numbered cycle $2 \mathrm{~N}$ $[4,5]$. Hereafter, we call this formulation the GO1 effect.

(b) The total sum of the sunspot number over the even cycle $2 N$ (intensity of the cycle $2 N$ ) has a good positive correlation with the intensity of succeeding odd cycle $2 N+1$, while correlation between corresponding intensities of the cycles $2 N-1$ and $2 N$ is weak. This is the original formulation of Gnevyshev and Ohl [13]. Here, this formulation is called the GO2 effect.

The Waldmeier relation [14] has two manifestations.

(a) A negative correlation between the duration of the ascending phase of a cycle $\tau$ and its amplitude. The $\tau$ value is defined as the period between the peak in the sunspot number of a cycle and the time elapsed from the minimum to reaching that peak. We call this effect W1.

(b) A positive correlation between the rise rate of a cycle $V$ and its amplitude [8]. We estimated the rise rate following Karak and Choudhuri [8], that is, by determining the difference between two points separated by one year, with the first point one year after the sunspot minimum. We call this effect W2. tions.

The amplitude-period relation has also two manifesta-

(a) A negative correlation between the amplitude of the solar cycle and its length $\Delta t$. This relation was mentioned in the works of Dicke [15], FriisChristensen and Lassen [16], Hoyng [17]. Hereafter we call this effect AP1. The $\Delta t$ value is defined by the time interval between subsequent solar cycle minima.

(b) A negative correlation between the amplitude of the solar cycle $N$ and the length of the previous cycle $N-1$ as measured from minima to minima. This link has been reported by Chernosky [18] and Wilson, Hathaway \& Reichmann [19]. We call this effect AP2.

\section{Datasets}

The solar indices used in analyses in this work are shown in Figure 1.

The data on sunspot number $R_{Z}$ and $R_{G}$ are taken

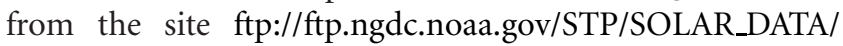
SUNSPOT_NUMBERS/. The data on sunspot area $\mathrm{Ar}$ are taken from http://www.gao.spb.ru/database/esai/. The majority of parameters needed for our analysis, including amplitudes of cycles, intensities of cycles, durations of ascending phases $(\tau)$, rise rates $(\nu)$, and durations of cycles $(\Delta t)$, were determined using 13 point average for monthly data. 


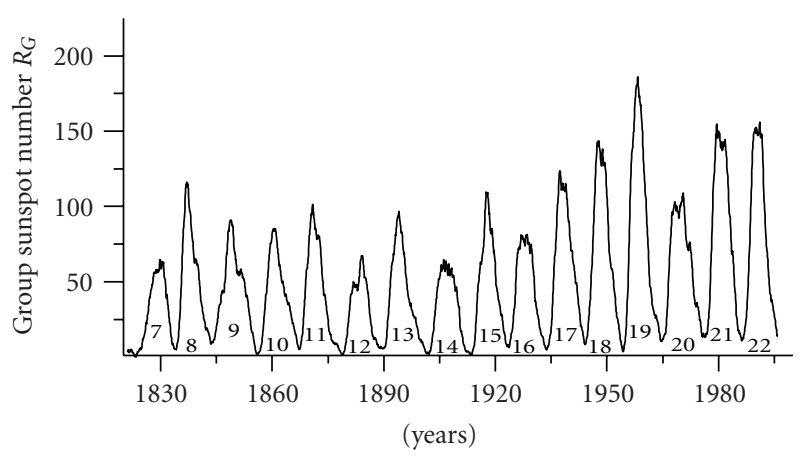

(a)

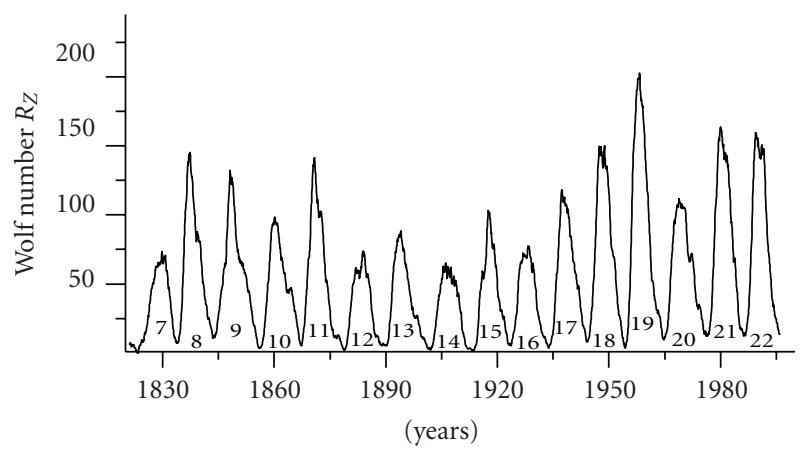

(b)

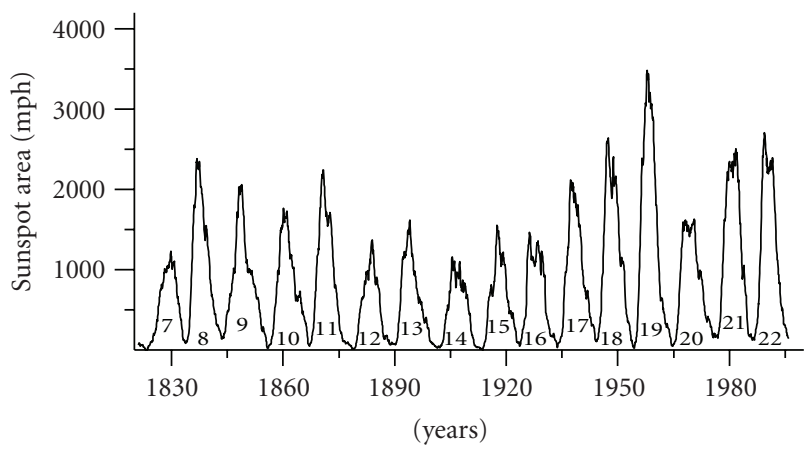

(c)

FIGURE 1: (a) group sunspot number; (b) Wolf sunspot number; (c) sunspot area in millionth of visible hemisphere. All the curves are 13-point averages of monthly data. Cycles are numbered according to Zürich system.

\section{Comparisons of Statistical Characteristics of Cycles in Different Solar Indices}

Statistical correlations between parameters of various manifestations of solar cycles and different solar indices are summarized in Table 1 and shown in Figures 1 and 2.

The significance of the correlation coefficients $R_{l}$, calculated above for all the 16 cycles, was estimated by means of the statistical test suggested by Ebisuzaki [20]. The statistical test is based on generating a large number of random series with the same power spectra (periodogram) as the first series but with random phases in the modes of the Fourier transform. The statistical significance of the original correlation was determined by comparing this correlation to the distribution of correlations between the randomphase series and the second original series. The significance levels are shown in Table 1 in italics (figures in brackets). Theoretical evaluation performed by another method, using Student's $t$-distribution of the value $t=r \sqrt{(n-2) /\left(1-r^{2}\right)}$ with $n-2$ degrees of freedom ( $n$ is a number of points), gives the similar results.

In Figures 2, 3 and 4, the best linear fits of the data are shown with solid lines and 1 standard deviation from the linear fits are shown with dotted lines. Figures 2-4 and Table 1 demonstrate that the GO2 and W2 effects are the most robust features of the solar cycles. They appear unambiguously in all of the solar indices, including spot area. The corresponding relationships are well described with linear functions, and correlation coefficients are significant at more than 0.99 confidence level. Our results for W2 effect are in agreement with the results of Cameron and Schüssler [7] and Karak and Choudhuri [8]. Cameron and Schüssler [7] have analyzed four datasets: monthly Wolf sunspot numbers for cycles 7-23, monthly group sunspot numbers for cycles $7-22$, sunspot areas for cycles $12-23$, and $10.7-\mathrm{cm}$ solar radio flux for cycles 19-23. In all the cases, they obtained high $\left(R_{l}=0.83-0.89\right)$ coefficient of correlation between rise rate and cycle amplitude. Karak and Choudhuri [8] found out that the corresponding correlation coefficients are even higher $\left(R_{l}=0.92-0.95\right)$ for Wolf number and sunspot area through the cycles $12-23$.

However, the integral even-odd effect GO2 was estimated using only seven points. Seven pairs of cycles is rather a limited number for drawing a decisive conclusion. However, using sunspot records $R_{Z}$ and $R_{G}$, we can examine GO2 relations over a far longer time interval. Figure 5 shows GO2 relationships for the sunspot cycles -4-22 (1700-1996).

It is evident from Figure 5 that in spite of lower quality of sunspot data before 1823, the integral Gnevyshev-Ohl rule does work quite plausibly through 1700-1996 (13 pairs of cycles). Only the pair of cycles 4-5 (Hale cycle 3 ) drops out of the even-odd correlation. With the exception of this one pair, the correlation coefficients $R_{l}$ exceed 0.90 and are significant at more than 0.99 confidence level. That is in agreement with the result of Nagovitsyn et al. [21] who analyzed GO2 effect in the reconstructed sunspot area over the last 400 years and obtained the corresponding correlation coefficient 0.907. It is remarkable that just for the cycles $4-5$, we have the least reliable sunspot data. Particularly, the years 1790-1794 were very poorly covered by sunspot observations, which were most likely due to the unstable political situation in Europe during that time [22]. That is why Usoskin et al. [23] hypothesized that cycle 4 was actually double and one small cycle has been lost in the 1790s, because of sparse and unreliable observations. An inclusion of the new cycle 1793-1800 in sunspot number records restores the order of the GO2 rule, making it valid over the last 400 years $[21,22]$.

We found out that the GO1 effect in both sunspot number records is only weakly significant prior to the year 1823. This might be a result of less precision in the sunspot data during 1700-1822, since the amplitude effect should be more sensitive to the data accuracy than the integral one. 
TABLE 1: Comparison of common properties of the six statistical effects (GO1, GO2, W1, W2, AP1, and AP2) with respect to group sunspot number, wolf number, and total sunspot area. Cycles span the time interval 1823-1996 (cycles 7-22).

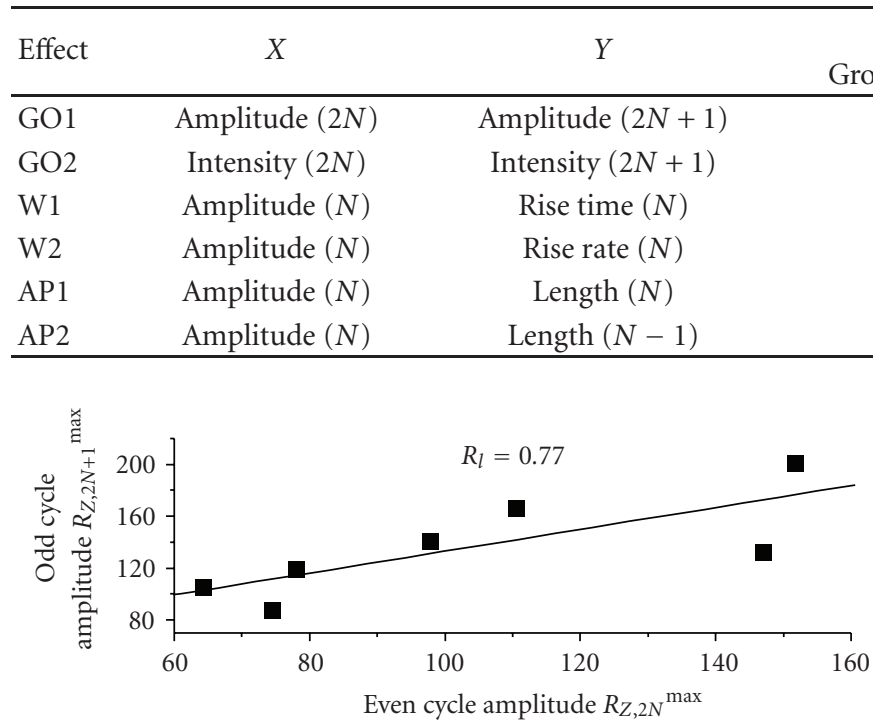

(a)

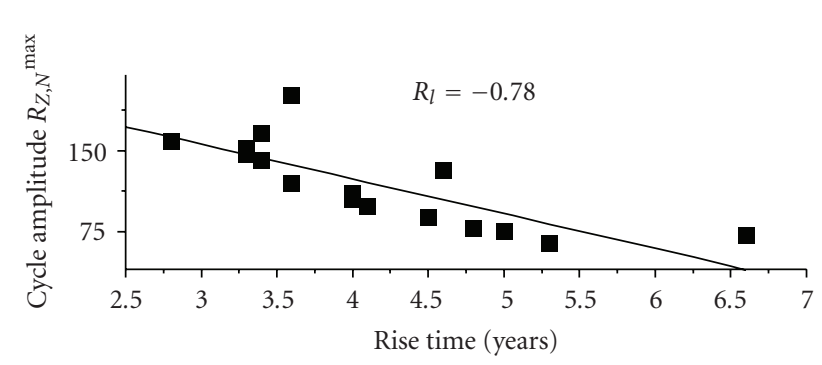

(c)

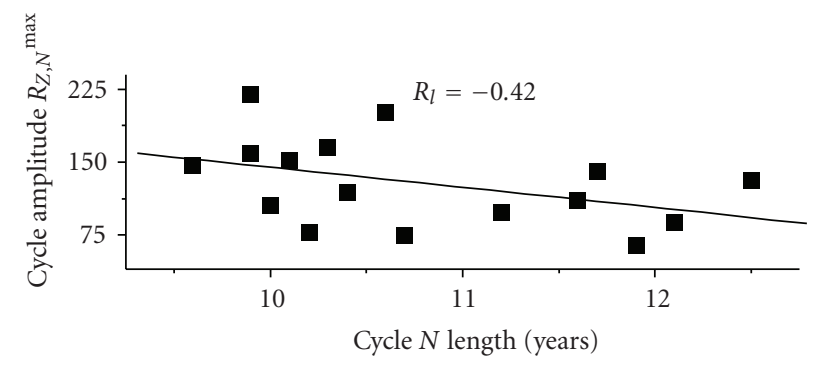

(e)
Coefficient of correlation between $X$ and $Y$

\begin{tabular}{ccc} 
Group sunspot number & Wolf number & Total sunspot area \\
\hline $0.69(0.92)$ & $0.77(0.97)$ & $0.77(0.98)$ \\
$0.93(0.9925)$ & $0.93(>0.9999)$ & $0.91(0.9965)$ \\
$-0.49(0.94)$ & $-0.78(0.9996)$ & $-0.43(0.89)$ \\
$0.86(0.9999)$ & $0.87(>0.9999)$ & $0.85(>0.9999)$ \\
$-0.53(0.96)$ & $-0.42(0.89)$ & $-0.28(0.73)$ \\
$-0.36(0.81)$ & $-0.48(0.93)$ & $-0.43(0.89)$ \\
\hline
\end{tabular}

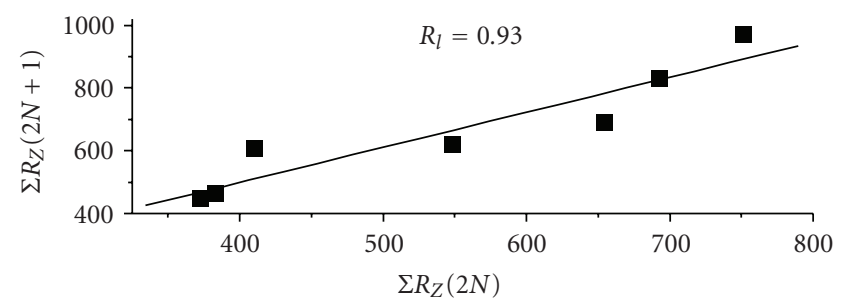

(b)

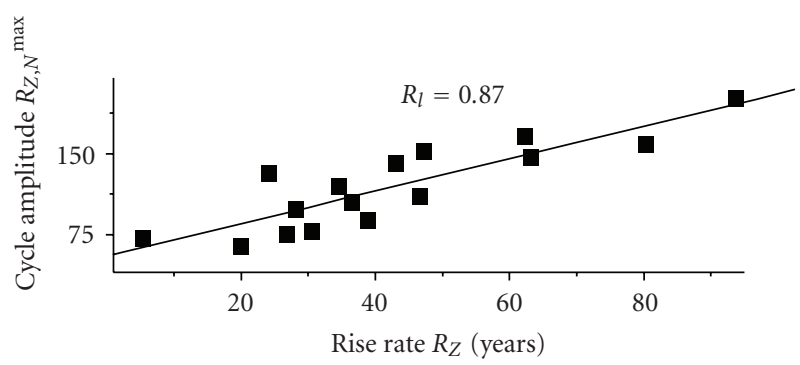

(d)

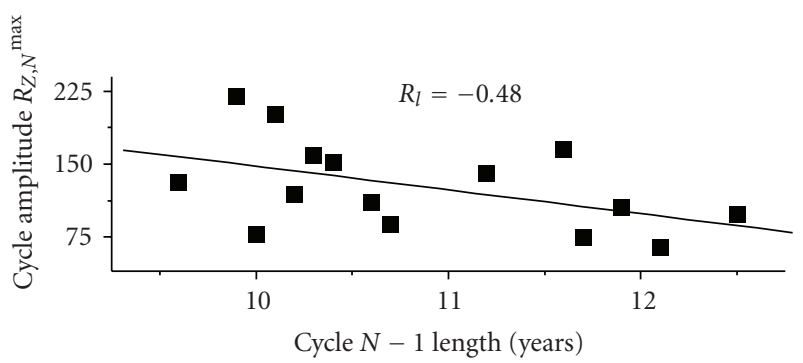

(f)

Figure 2: Different statistical effects in Wolf number cycles 7-22: (a) Gnevyshev-Ohl GO1; (b) Gnevyshev-Ohl GO2; (c) Waldmeier W1; (d) Waldmeier W2; (d) amplitude-period AP1; (f) amplitude-period AP2. A standard deviation from the linear fit is shown with dotted lines.

\section{Discussion and Conclusion}

We examined six statistical features (GO1, GO2, W1, W2, AP1, and AP2 effects) in 16 cycles (1823-1996) in solar indices $R_{Z}, R_{G}$, and $A r$, including the extended sunspot area series of the Pulkovo observatory. The analyses showed that two statistical effects are the most significant.

(a) a positive correlation between intensity of the cycle $2 N$ and intensity of the cycle $2 N+1$ (GO2 effect). (b) a positive correlation between the rise rate of a cycle and its strength (W2 effect).

These two effects are evident in all the data, including both statistical sunspot indices $R_{Z}$ and $R_{G}$ as well as sunspot area index, which has fairly defined physical meaning. The corresponding correlations are significant at more than 0.99 confidence level. In sunspot number, the GO2 relation exists throughout the entire time interval 1700-1996.

Our results, obtained for GO2 and W2 effects, are in agreement with the results of other authors who used 


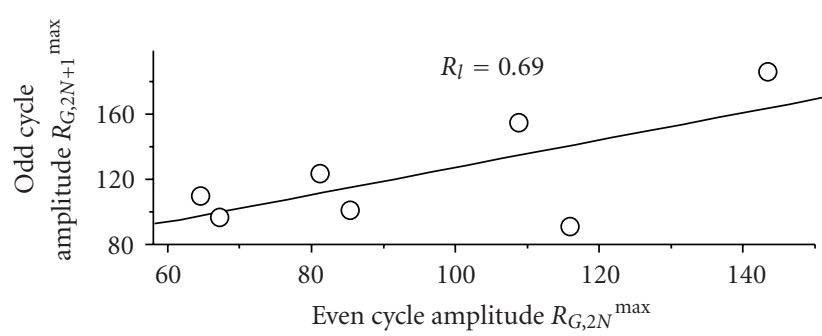

(a)

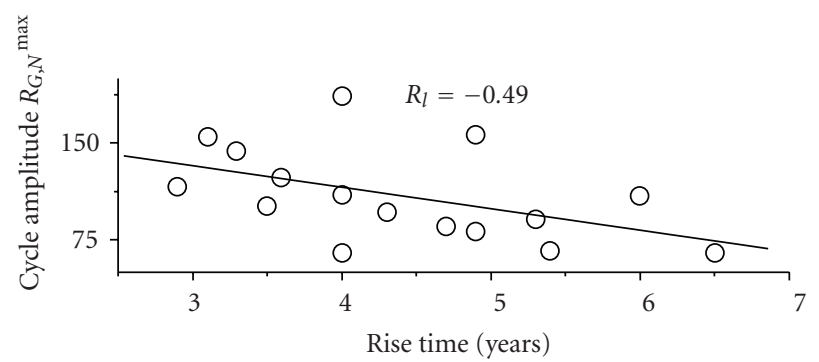

(c)

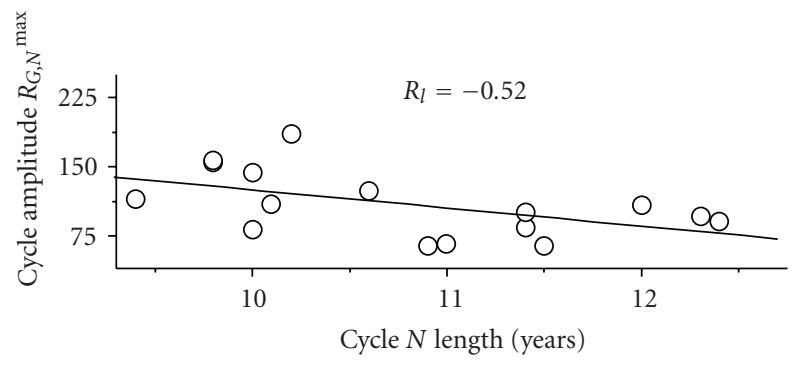

(e)

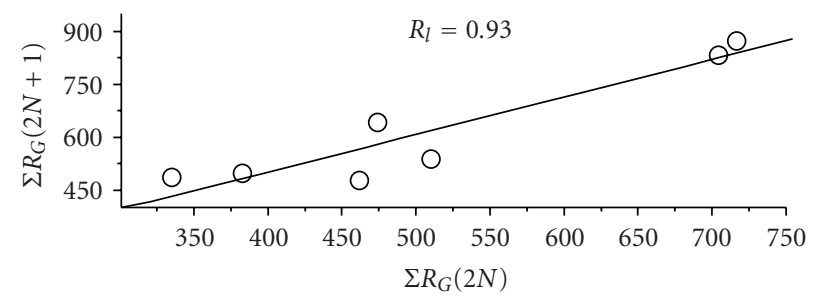

(b)

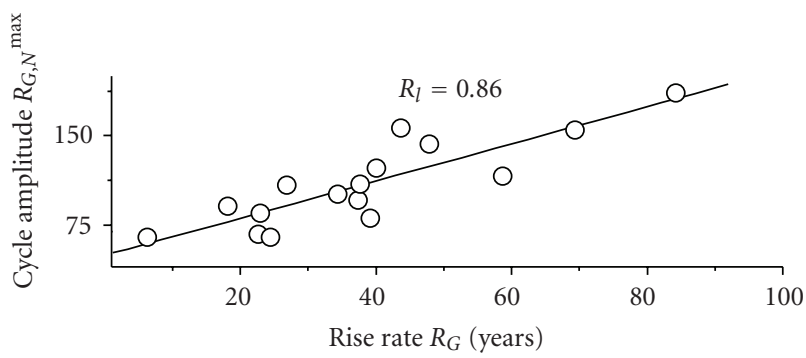

(d)

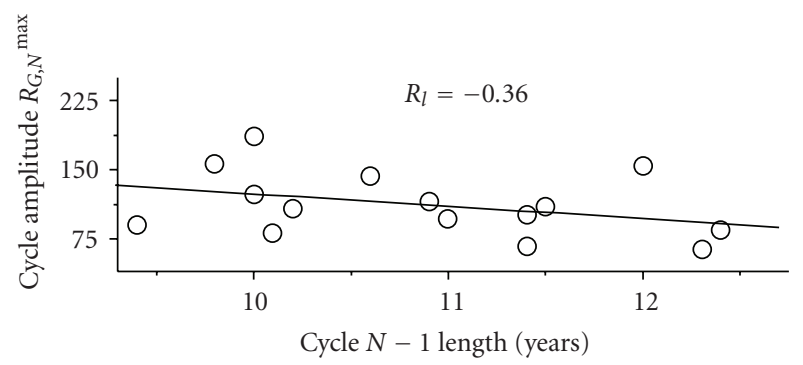

(f)

FIGURE 3: Different statistical effects in group sunspot number cycles 7-22: (a) Gnevyshev-Ohl GO1; (b) Gnevyshev-Ohl GO2; (c) Waldmeier W1; (d) Waldmeier W2; (e) amplitude-period AP1; (f) amplitude-period AP2. A standard deviation from the linear fit is shown with dotted lines.

(a) different time intervals (b) different ways of the cycle parameters determination and sometimes other solar proxies. That shows that GO2 and W2 relations are robust relative to change of the solar data set and methods of its analysis and reflect the actual properties of the solar activity.

The amplitude of even-odd effect GO1 is confirmed in all the solar series during 1823-1996 with the confidence significant at more than 0.90 level. In the sunspot records, the GO1 relation is less pronounced before 1823, which might be connected with lesser accuracy of the data.

The rest of the effects (W1, AP1, and AP2) are obviously not manifested together in all the three solar indices over 1823-1996. Furthermore, only little evidence for the relations W1, AP1, AP2 were found in sunspot area data. Our result for W1 effect in sunspot area $\left(R_{l}=-0.43\right)$ is in fairly agreement with the result of Karak and Choudhuri [8] $\left(R_{l}=-0.31\right.$ for the cycles $\left.12-23\right)$ but apparently differ from the result of Dikpati et al. [6]. The most likely cause of the disagreement is difference in the methods used for the rise time determination.
Thus, the analyses of the available solar data, including both statistical and physical indices, make it possible to assume that the integral even-odd effect and the rise rateamplitude effect are the most prominent and universal statistical features of the solar activity cycles. Other statistical effects (W1, AP1, and AP2) are sensitive to selection of solar index, interval of analysis, and way of the cycle parameter determination. The stability of the GO2 relation confirms the result of Nagovitsyn et al. [21], who concluded that the GO2 rule could be considered as a rather strict principle of longterm solar magnetic field dynamics. The principle means that the 22-year cycle present in solar magnetic activity. Each 22year cycle arises as a consequence of a pair of consecutive 11year cycles and begins the even sunspot cycle according to the Zürich numbering.

In spite of some rather interesting lines of reasoning suggested by Durney [24] Usoskin and Mursula [22] Benevolenskaya [25], the actual physical nature of the cycle pairing is not clearly understood, and this phenomenon require further research. The stability of the W2 relationship is in agreement with the theoretical estimations of Karak and 


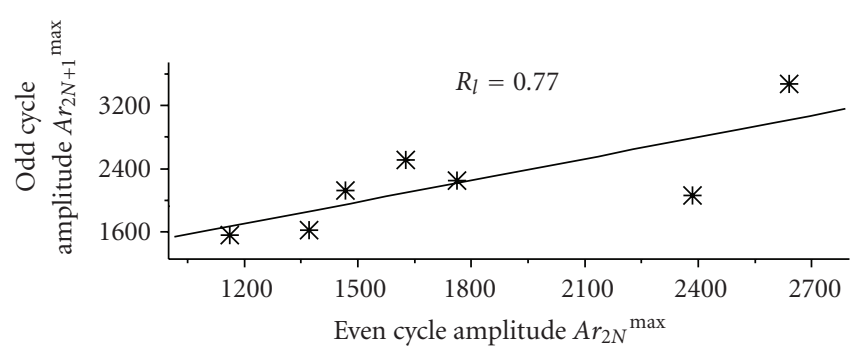

(a)

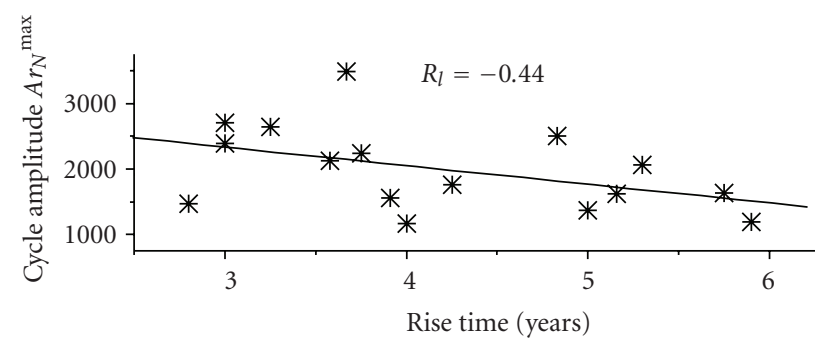

(c)

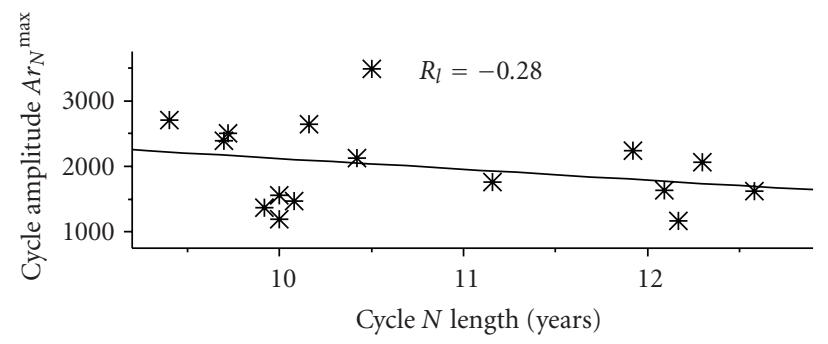

(e)

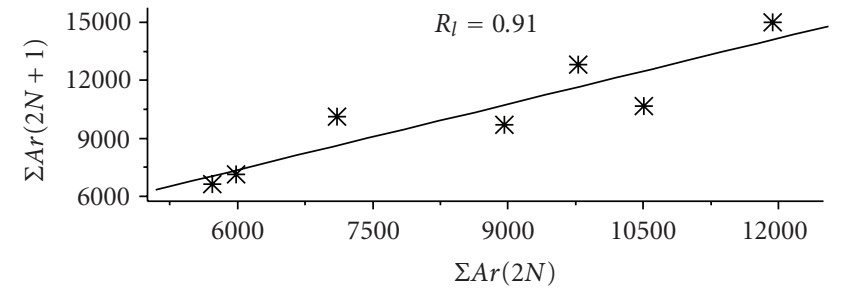

(b)

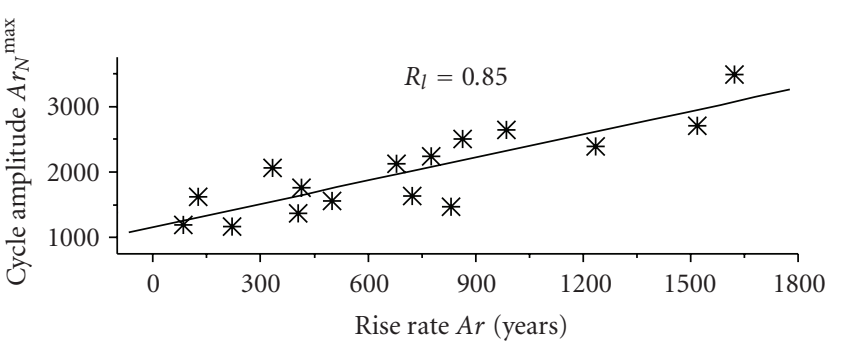

(d)

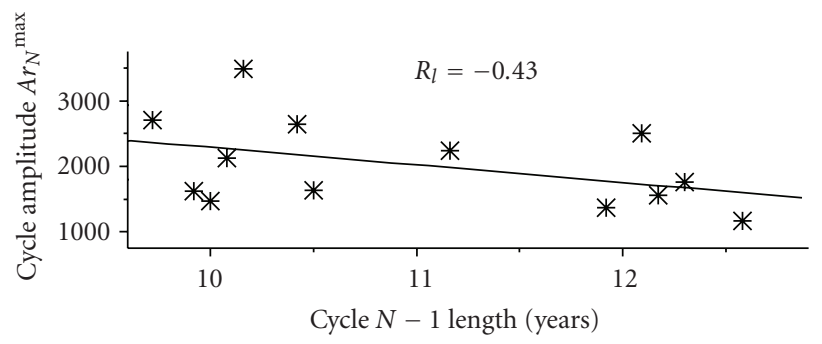

(f)

FIgure 4: Different statistical effects in sunspot area cycles 7-22: (a) Gnevyshev-Ohl GO1; (b) Gnevyshev-Ohl GO2; (c) Waldmeier W1; (d) Waldmeier W2; (e) amplitude-period AP1; (f) amplitude-period AP2. A standard deviation from the linear fit is shown with dotted lines.

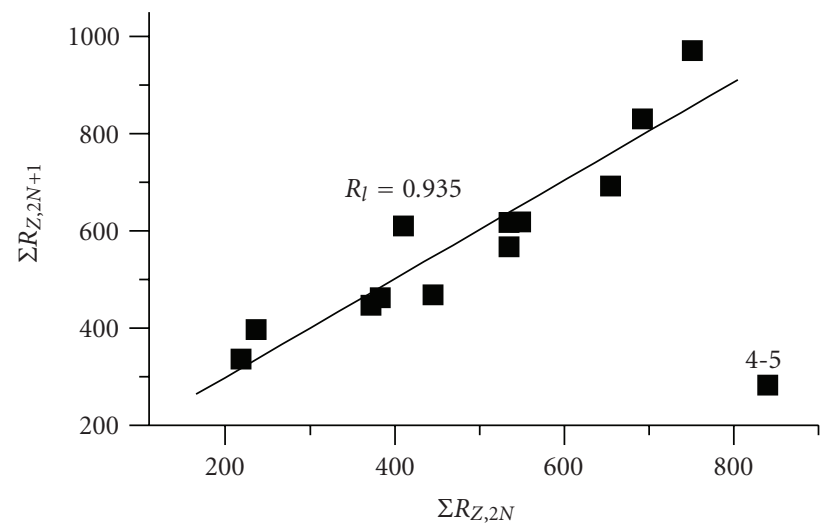

(a)

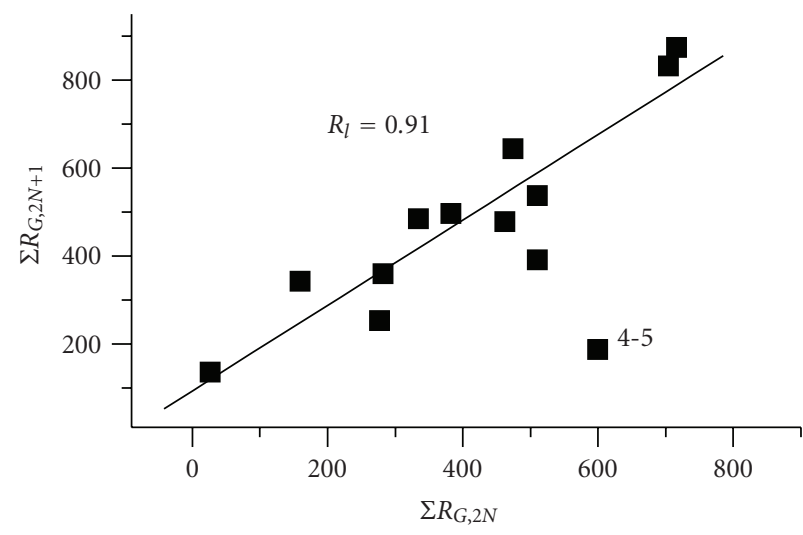

(b)

FIGURE 5: GO2 effect in sunspot number cycles -4-22: (a) Wolf number; (b) group sunspot number. Linear trends and correlation coefficients are calculated using all other points except pair of cycles 4-5.

Choudhuri [8], who found that the effect W2 is very robust and is reproduced easily in different types of dynamo models. It is evident that both GO2 and W2 effects should be taken into account in solar activity forecasts as well as dynamo modeling.

\section{Acknowledgments}

M. Ogurtsov expresses his thanks to the exchange program between the Russian and Finnish Academies (Project no. 16), program of the Saint-Petersburg Scientific Center of 
RAS for 2011, and RFBR Grants nos. 07-02-00379, 09-0200083, 10-05-00129, and 11-02-00755 for financial support. M. Lindholm was supported by the Commission of EU (project Millennium, Contract no. 017008).

\section{References}

[1] R. Schwenn, "Space weather: the solar perspective," Living Reviews in Solar Physics, vol. 3, p. 2, 2006.

[2] T. Pulkkinen, "Space weather: terrestrial perspective," Living Reviews in Solar Physics, vol. 4, p. 1, 2007.

[3] Yu. I. Vitinsky, M. Kopecky, and G. V. Kuklin, Statistics of Sunspot Activity, Nauka, Moscow, Russia, 1986.

[4] P. Charbonneau, "Multiperiodicity, chaos, and intermittency in a reduced model of the solar cycle," Solar Physics, vol. 199, no. 2, pp. 385-404, 2001.

[5] D. H. Hathaway, R. M. Wilson, and E. J. Reichmann, "Group sunspot numbers: sunspot cycle characteristics," Solar Physics, vol. 211, no. 1-2, pp. 357-370, 2002.

[6] M. Dikpati, P. A. Gilman, and G. De Toma, "The waldmeier effect: an artifact of the definition of wolf sunspot number?" Astrophysical Journal, vol. 673, no. 1, pp. L99-L101, 2008.

[7] R. Cameron and M. Schüssler, "A robust correlation between growth rate and amplitude of solar cycles: consequences for prediction methods," Astrophysical Journal, vol. 685, no. 2, pp. 1291-1296, 2008.

[8] B. B. Karak and A. R. Choudhuri, "The Waldmeier effect and the flux transport solar dynamo," Monthly Notices of the Royal Astronomical Society. vol. 410, pp. 1503-1512, 2011.

[9] D. V. Hoyt and K. H. Schatten, "Group sunspot numbers: a new solar activity reconstruction," Solar Physics, vol. 179, no. 1, pp. 189-219, 1998.

[10] Yu. A. Nagovitsyn, "To the description of long-term variations in the solar magnetic flux: the sunspot area index," Astronomy Letters, vol. 31, pp. 557-562, 2005.

[11] Y. A. Nagovitsyn, V. G. Ivanov, E. V. Miletsky, and D. M. Volobuev, "ESAI database and some properties of solar activity in the past," Solar Physics, vol. 224, no. 1-2, pp. 103-112, 2005.

[12] J. A. Eddy, "The maunder minimum," Science, vol. 192, no. 4245, pp. 1189-1202, 1976.

[13] M. N. Gnevyshev and A. I. Ohl, "On the 22-year cycle in solar activity," Astronomy Reports, vol. 25, pp. 18-20, 1948, Russian.

[14] M. Waldmeier, "Neue Eigenschaften der Sonnenflecken kurve (New qualities of the sunspot curves)," Astronomische Mitteilungen Zürich, vol. 14, pp. 105-130, 1935.

[15] R. H. Dicke, "Is there a chronometer hidden deep in the sun?" Nature, vol. 276, no. 5689, pp. 676-680, 1978.

[16] E. Friis-Christensen and K. Lassen, "Length of the solar cycle: an indicator of solar activity closely associated with climate," Science, vol. 254, no. 5032, pp. 698-700, 1991.

[17] P. Hoyng, "Helicity fluctuations in mean field theory: an explanation for the variability of the solar cycle?" Astronomy \& Astrophysics, vol. 272, pp. 321-329, 1993.

[18] E. J. Chernosky, "A relationship between the length and activity of sunspot cycles," Publications of the Astronomical Society of the Pacific, vol. 66, p. 241, 1954.

[19] R. M. Wilson, D. H. Hathaway, and E. J. Reichmann, "Estimating the size and timing of maximum amplitude for cycle 23 from its early cycle behavior," Journal of Geophysical Research, vol. 103, pp. 17411-17418, 1998.

[20] W. Ebisuzaki, "A method to estimate the statistical significance of a correlation when the data are serially correlated," Journal of Climate, vol. 10, no. 9, pp. 2147-2153, 1997.
[21] Y. A. Nagovitsyn, E. Y. Nagovitsyna, and V. V. Makarova, "The Gnevyshev-Ohl rule for physical parameters of the solar magnetic field: the 400-year interval," Astronomy Letters, vol. 35, no. 8, pp. 564-571, 2009.

[22] I. G. Usoskin and K. Mursula, "Long-term solar cycle evolution: review of recent developments," Solar Physics, vol. 218, no. 1-2, pp. 319-343, 2003.

[23] I. G. Usoskin, K. Mursula, and G. A. Kovaltsov, "Lost sunspot cycle in the beginning of Dalton minimum: new evidence and consequences," Geophysical Research Letters, vol. 29, no. 24, pp. 36-1, 2002.

[24] B. R. Durney, "On the differences between odd and even solar cycles," Solar Physics, vol. 196, no. 2, pp. 421-426, 2000.

[25] V. Benevolenskaya, "Is the solar cycle still a puzzle?" in Proceedings of the IAU Symposium 233, V. Bothmer and A. A. Hady, Eds., pp. 215-221, Cambridge University Press, Cairo, Egypt, March-April 2006. 

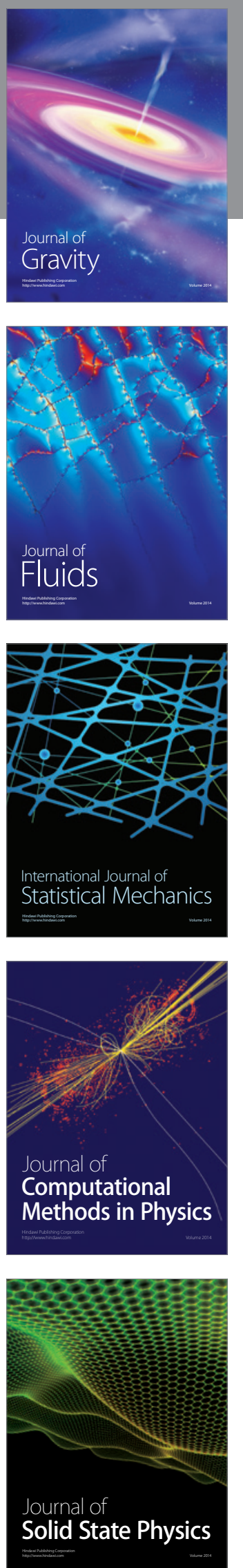

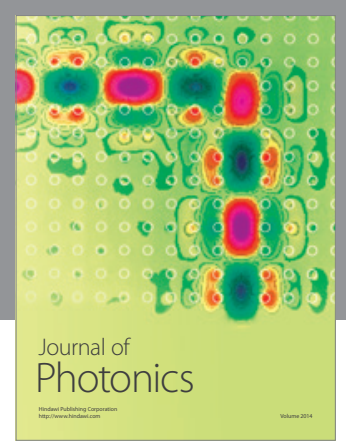

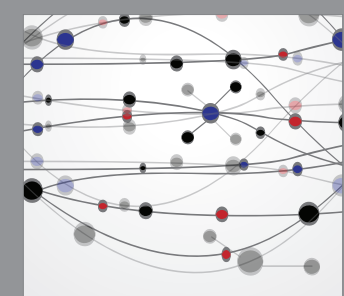

The Scientific World Journal
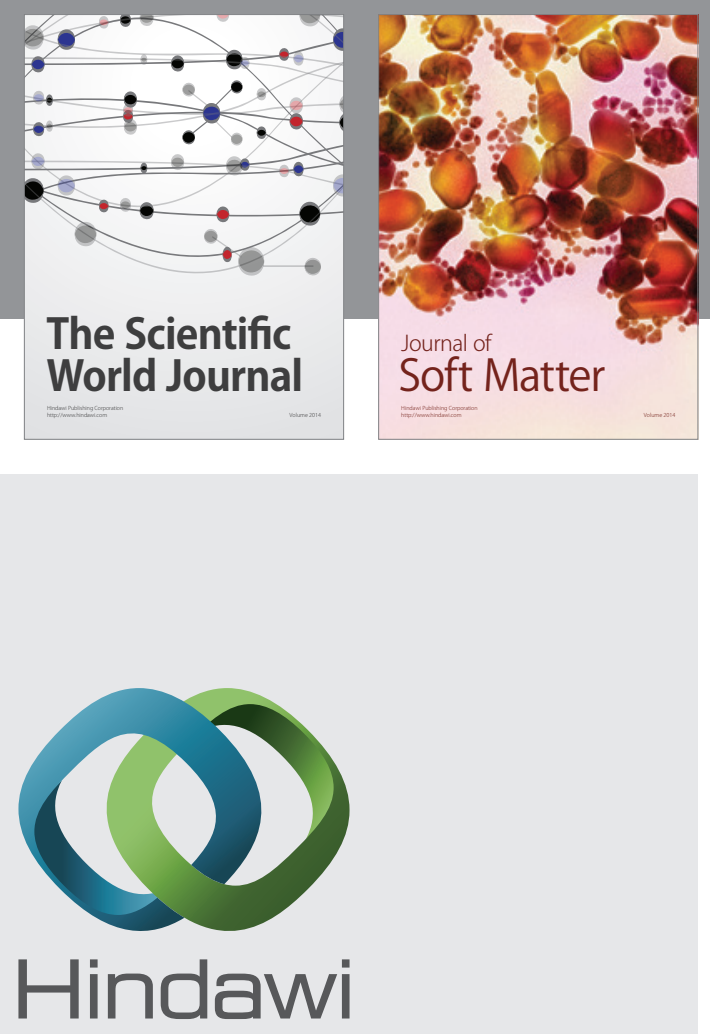

Submit your manuscripts at

http://www.hindawi.com
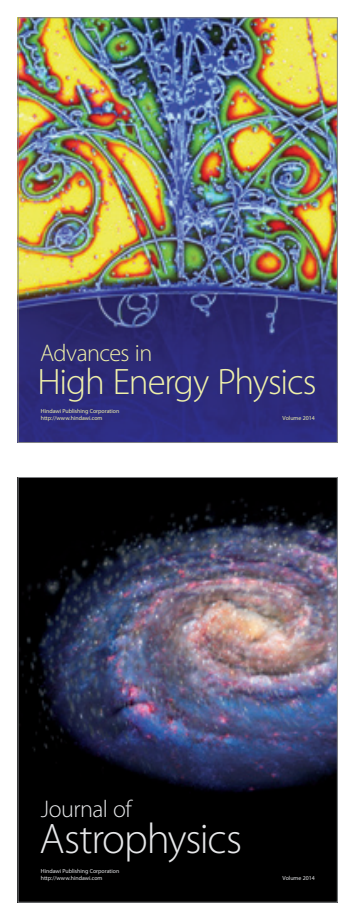
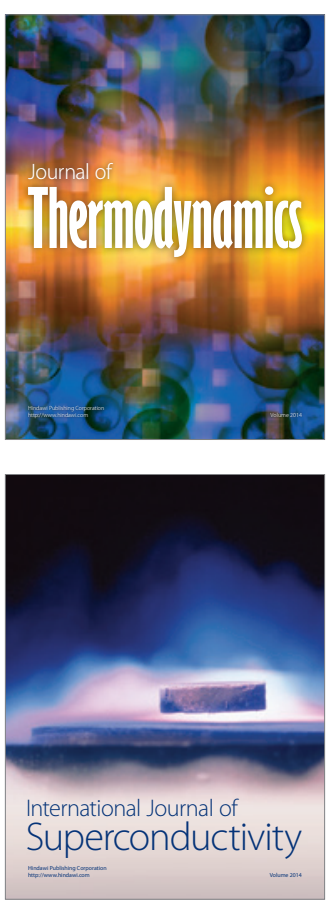
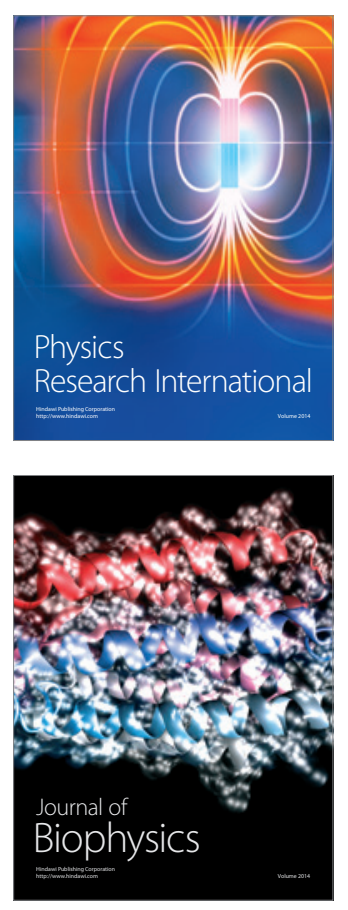
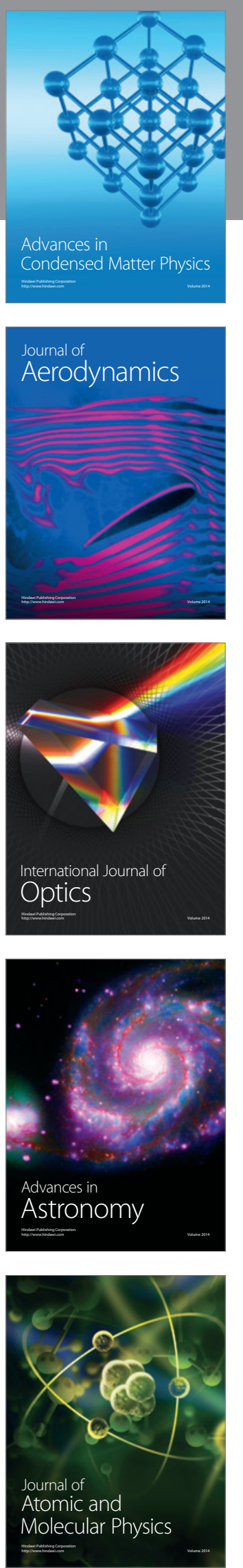